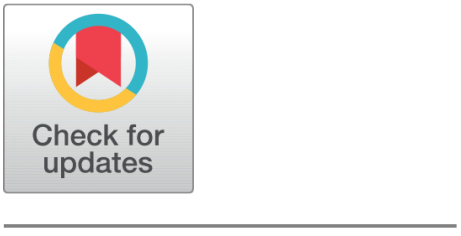

open ACCESS

Received: 15.09 .2020

Accepted: 04.02.2021

Published: 12.02 .2021

Citation: Meenapriya P, Uma Maheswari K, Ratchagar NP (2021) Concentration of air pollutants in an inclined channel with the effect of electric and magnetic field. Indian Journal of Science and Technology 14(5): 393-405. https://doi.org/ 10.17485/IJST/v14i5.1661

* Corresponding author. umamohansusd@gmail.com

Funding: None

Competing Interests: None

Copyright: ( 2021 Meenapriya et al. This is an open access article distributed under the terms of the Creative Commons Attribution License, which permits unrestricted use, distribution, and reproduction in any medium, provided the original author and source are credited.

Published By Indian Society for Education and Environment (iSee)

ISSN

Print: 0974-6846

Electronic: 0974-5645

\section{Concentration of air pollutants in an inclined channel with the effect of electric and magnetic field}

\author{
P Meenapriya ${ }^{1}$, K Uma Maheswari ${ }^{1}{ }^{*}$, Nirmala P Ratchagar ${ }^{1}$ \\ 1 Department of Mathematics, Annamalai University, Chidambaram, 608 002, India
}

\section{Abstract}

Background/Objectives: Atmospheric visibility is predominantly affected by aerosols and it is improved by reducing the effects of concentration of smog and haze. The model is developed suitably to study the effects of smog and haze through the concentration of aerosols in the presence and absence of chemical reactions. Methods: The atmospheric fluid in the inclined channel flow is assumed to be incompressible, laminar, and presented through the solution of Navier Stokes equation for velocity and advectiondiffusion equation for the concentration of aerosols. The governing equations of the study are attained as two-dimensional partial differential equations and are solved using the perturbation technique with appropriate boundary conditions. The solution leads to detailed graphs for velocity components and concentration of aerosols. Findings: This study examined the effects of concentration of smog and haze semi analytically in a two-dimensional channel bounded by porous beds together with the effects of the electric and magnetic field. The derived results are depicted graphically for different parameters. The obtained results of concentration clearly show that there is no considerable influence of porous, gravity and magnetic field effect in reducing smog and haze concentration, whereas the increasing electric field reduces the haze concentration.

Keywords: Aerosols; Darcy law; haze; perturbation method; porous beds; smog

\section{Introduction}

Literature in the field of atmospheric research focuses on aerosol particles and their contribution to air pollution. The concentration of air pollutants from an urban source in the presence of mesoscale wind is analyzed and results are presented in ${ }^{(1)}$. The significance of aerosols in the Earth system and the development of aerosol sciences and engineering is presented in ${ }^{(2)}$. Many analytical approaches are listed to study the mechanisms of aerosols and the behavior of dust particles in electric and magnetic fields ${ }^{(3,4)}$.

The elliptic and hyperbolic problems using perturbation techniques are investigated and showed how the method tends to combine the behavior of a variety of problems 
in perturbation theory ${ }^{(5)}$. The basic perturbation technique which is used for much research work is presented in ${ }^{(6)}$.

Basically, the inclined geometry has vast applications in realistic practical situations. The heat transfer and convective flow in an inclined channel bounded by two rigid plates held at different temperatures with two regions using the regular perturbation method are studied analytically ${ }^{(7)}$. The results for particle deposition in the turbulent inclined channel in the presence and absence of thermophoresis are discussed ${ }^{(8)}$. In ${ }^{(9)}$ the convective Couette flow of a Jeffrey fluid in an inclined channel when the walls are provided with porous lining is studied. The peristaltic flow of a couple stress fluid in a two-dimensional inclined channel with magnetic effect by Adomian decomposition method ${ }^{(10)}$.

Numerous publications have investigated the combined effects of the magnetic field, permeable walls, Darcy velocity, electrically conducting fluid, couette flow. Analytical solutions for the hydromagnetic flow of Newtonian fluid flowing in a uniform channel bounded by porous beds were studied in ${ }^{(11)}$. The magnetic effects of electrically conducting two-fluid between two inclined channels in a rotating system are examined in ${ }^{(12)}$.

Several studies are existing in the field of atmospheric pollution and specifically aerosols. But the analytical approach of aerosol study is limited that too two-dimensional study are very few. The above studies are encouraging for developing a twodimensional model to focus the effects of smog and haze by calculating the concentration of aerosols with and without chemical reaction in the atmospheric fluid in an inclined channel. Numerical calculations are computed by MATHEMATICA and the results are portrayed graphically which helps to analyse the effects of concentration of aerosols. Result reveals that the electric field plays a vital role than a magnetic field in an inclined flow of atmospheric aerosols.

\section{Mathematical Formulation}

A study of the viscous incompressible flow of atmospheric fluid flowing steadily downstream in an open infinitely long inclined under gravity is designed. Assuming the atmospheric fluid understudy is poorly conducting. The geometry of the system considered in this present study is sketched in Figure 1. It depicts that $\mathrm{x}$-axis is taken parallel to the inclined channel while the $y$ - direction is taken normal to it. The channel is symmetric about $x$-axis and inclined at an angle $\theta$ with the horizontal bounded by porous layers on both sides.

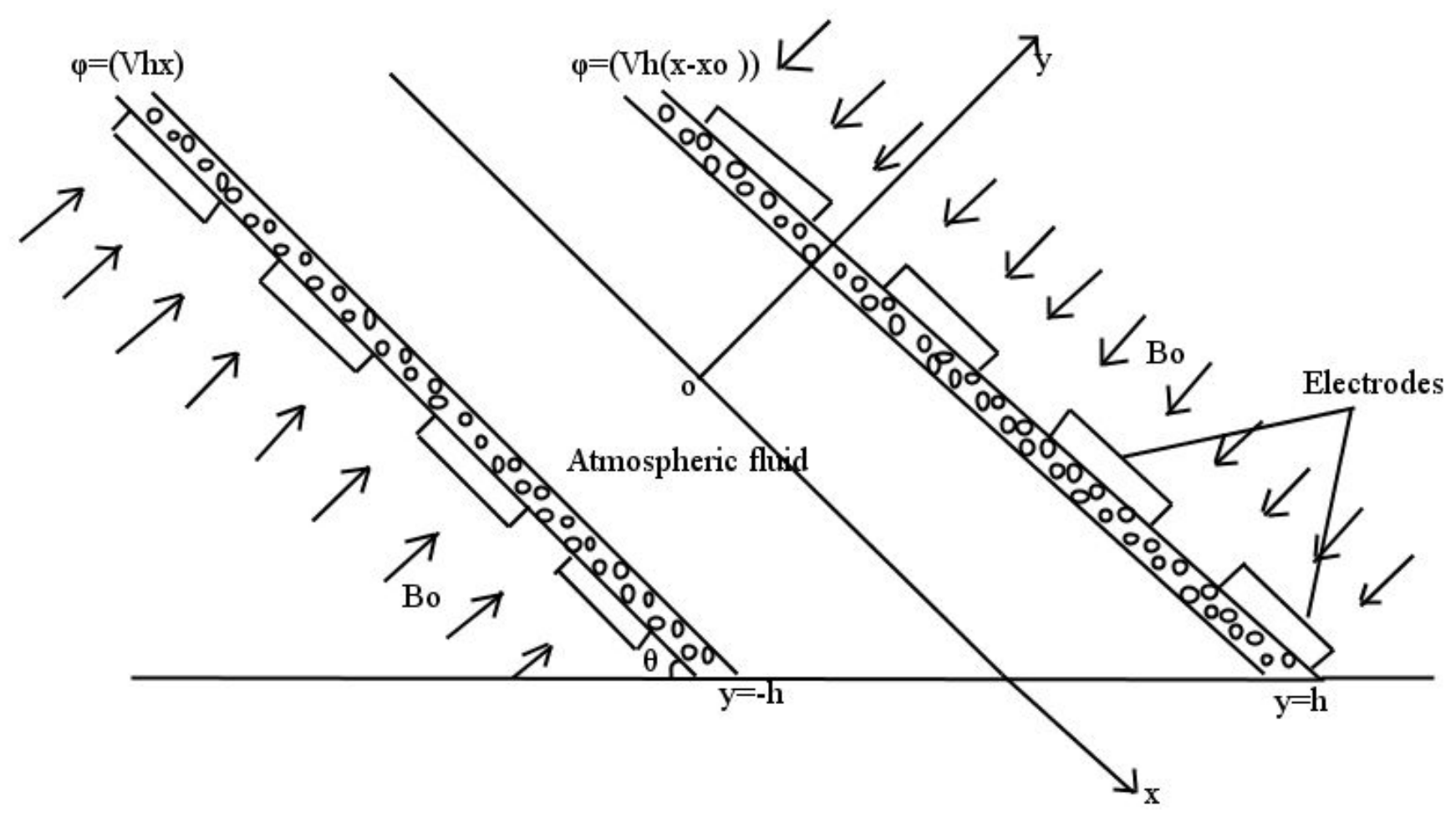

Fig 1. Physical configuration

The electric and uniform magnetic field is applied transverse to the system externally through the boundaries. The electric field is applied through electrodes which are electro-conducting impermeable rigid plates with electric potentials 
$\varphi=(V h x)$ at $y=-h, \varphi=\left(V h\left(x-x_{0}\right)\right)$ at $y=h$. A strong transverse magnetic field of unit strength $B_{0}$ is applied externally, neglecting the induced electric and magnetic field due to low Reynolds number.

The fluid is assumed to be driven by the laminar flow with a constant pressure gradient. Based on all the assumptions made above, the governing equations are as follows.

Continuity equation

$$
\frac{\partial u}{\partial x}+\frac{\partial v}{\partial y}=0
$$

$\mathrm{x}$ - Momentum equation

$$
\rho\left[\frac{\partial u}{\partial t}+u \frac{\partial u}{\partial x}+v \frac{\partial u}{\partial y}\right]=-\frac{\partial p}{\partial x}+\mu\left(\frac{\partial^{2} u}{\partial x^{2}}+\frac{\partial^{2} u}{\partial y^{2}}\right)+\rho_{e} E_{x}-\sigma_{c} u B_{0}^{2}+\rho g \sin \theta
$$

$\mathrm{y}$ - Momentum equation

$$
\rho\left[\frac{\partial v}{\partial t}+u \frac{\partial v}{\partial x}+v \frac{\partial v}{\partial y}\right]=\mu\left(\frac{\partial^{2} v}{\partial x^{2}}+\frac{\partial^{2} v}{\partial y^{2}}\right)-\rho g \cos \theta
$$

Species equation

$$
\left(\frac{\partial C_{i}}{\partial t}+u \frac{\partial C_{i}}{\partial x}+v \frac{\partial C_{i}}{\partial y}\right)=D\left(\frac{\partial^{2} C_{i}}{\partial x^{2}}+\frac{\partial^{2} C_{i}}{\partial y^{2}}\right)-K C_{i}
$$

Let $\mathrm{u}, \mathrm{v}$ be the velocity components in the direction of $\mathrm{x}$ and $\mathrm{y}$-axis respectively, $\rho$ is the density of the fluid, $g$ is the acceleration due to gravity, $\mu$ is the co-efficient of viscosity, $p$ is the pressure of the fluid, $E_{x}$ is the electric field, $B_{0}$ is applied magnetic field and $\rho_{e}$ is the density of charge distribution. Using perturbation technique the governing equations are solved assuming that homogeneous first-order chemical reaction $\mathrm{K}$ takes place inside the channel with $\mathrm{D}$ as the mass diffusivity.

The concentration of aerosols is represented by $C_{i}$ assuming $\beta \neq 0$ when $i=1$, and $\beta=0$ when $i=2$. In other words $C_{1}$ denotes the concentration of smog, while $C_{2}$ symbolises the concentration of haze. To solve the governing equations Beavers Joseph $^{(13)}$ slip conditions are used to describe the boundary effects for velocity.

$$
\begin{gathered}
u=0, v=0, \quad C_{i}=C_{0} \varepsilon e^{i(\alpha x+w t)}, \text { at } y=0 \\
\frac{\partial u}{\partial y}=\frac{-\alpha_{p}}{\sqrt{k}}\left(u-u_{p}\right), v=v_{1}, C_{i}=C_{0}\left(1+\varepsilon e^{i(\alpha x+w t)}\right), \text { at } y=h \\
\frac{\partial u}{\partial y}=\frac{\alpha_{p}}{\sqrt{k}}\left(u-u_{p}\right), v=v_{2}, \quad \text { at } y=-h
\end{gathered}
$$

Where $u_{p}$ is the Darcy velocity of the porous layer, $\alpha_{p}$ is the slip parameter, $k$ is the permeability of the porous layer, $\alpha$ is the streamwise wave number, $\mathrm{w}$ is the frequency parameter, $\varepsilon$ is the perturbation parameter, $\mathbf{i}$ is the imaginary number and $u_{p}=-\frac{k}{\mu} \frac{\partial p}{\partial x}$ represents Darcy law. To make (1) to (7) dimensionless the following dimensionless quantities are introduced.

$$
\begin{gathered}
x^{*}=\frac{x}{h} ; y^{*}=\frac{y}{h} ; u^{*}=\frac{u}{u_{0}} ; v^{*}=\frac{v}{u_{0}} ; \rho_{e}^{*}=\frac{\rho_{e}}{\left(\varepsilon_{0} \frac{V}{h^{2}}\right)} ; \\
p^{*}=\frac{p}{\rho u_{0}^{2}} ; t^{*}=\frac{t}{t_{0}} ; u_{p}^{*}=\frac{u_{p}}{u_{0}} ; E_{x}^{*}=\frac{E_{x}}{\left(\frac{V}{h}\right)} ; \beta^{2}=\frac{h^{2} K}{D} ; C_{i}^{*}=\frac{C_{i}}{C_{0}}
\end{gathered}
$$

The chemical reaction rate parameter is $\beta$ and $V$ is the applied constant electric potential due to embedded electrodes at the boundaries. The above governing equations and boundary conditions are made dimensionless and neglecting asterisk the following equations are obtained.

$$
\frac{\partial u}{\partial x}+\frac{\partial v}{\partial y}=0
$$




$$
\begin{aligned}
& \left(\frac{\partial^{2} u}{\partial x^{2}}+\frac{\partial^{2} u}{\partial y^{2}}\right)-\operatorname{Re}\left(a_{0} \frac{\partial u}{\partial t}+u \frac{\partial u}{\partial x}+v \frac{\partial u}{\partial y}-\frac{\partial p}{\partial x}\right)+W_{e} \rho_{e} E_{x}-u M^{2}+G \sin \theta=0 \\
& \frac{\partial^{2} v}{\partial x^{2}}+\frac{\partial^{2} v}{\partial y^{2}}-\operatorname{Re}\left(a_{0} \frac{\partial v}{\partial t}+u \frac{\partial v}{\partial x}+v \frac{\partial v}{\partial y}\right)-G \cos \theta=0 \\
& \left(\frac{\partial^{2} C_{i}}{\partial x^{2}}+\frac{\partial^{2} C_{i}}{\partial y^{2}}\right)-\left(a_{2} \frac{\partial C_{i}}{\partial t}+a_{1}\left(u \frac{\partial C_{i}}{\partial x}+v \frac{\partial C_{i}}{\partial y}\right)\right)-\beta^{2} C_{i}=0 \\
& u=0, v=0, C_{i}=\left(\in e^{i(\alpha x+w t)}\right), \text { at } y=0 \\
& \frac{\partial u}{\partial y}=-\alpha \sigma\left(u-u_{p}\right), v=v_{1}, C_{i}=\left(1+\in e^{i(\alpha x+w t)}\right) \text { at } y=1 \\
& \frac{\partial u}{\partial y}=\alpha \sigma\left(u-u_{p}\right), \quad v=v_{2}, \quad \text { at } y=-1
\end{aligned}
$$

Where the porous parameter $\sigma=\frac{h}{\sqrt{k}}$, the electric number $W_{e}=\frac{\left(\in_{0} V^{2}\right)}{\mu h u_{0}}$, the Hartmann number $M^{2}=\frac{\sigma_{0} B_{0}^{2} h^{2}}{\mu}$, the gravitational parameter $G=\frac{\rho g h^{2}}{\mu u_{0}}$, the Reynolds number $R e=\frac{\rho u_{0} h}{\mu}, a_{0}=\frac{h u_{0}}{t_{0}}, a_{1}=\frac{h u_{0}}{D}, b_{1}=\frac{h^{2}}{D t_{0}}$. Using Maxwell's equations and conservation of charges equation, the value of $\rho_{e} E_{x}$ is calculated as

$\rho_{e} E_{x}=a_{2} e^{-\alpha_{c} y}$, where $a_{2}=\frac{-\alpha_{c}^{2} X_{0} P_{e}}{2 \sinh \alpha_{c} h}$

so (9) takes the form,

$$
\left(\frac{\partial^{2} u}{\partial x^{2}}+\frac{\partial^{2} u}{\partial y^{2}}\right)-\operatorname{Re}\left(a_{0} \frac{\partial u}{\partial t}+u \frac{\partial u}{\partial x}+v \frac{\partial u}{\partial y}-\frac{\partial p}{\partial x}\right)+W e a_{2} e^{-\alpha_{c} y}-u M^{2}+G \sin \theta=0
$$

\subsection{Method of solution}

The perturbation technique is used to evaluate the values of velocity and concentration of air pollutants. Decomposing the flow variables into steady base state quantities denoted by upper case and two-dimensional linear perturbed quantities denoted by tilde $(\sim)$ symbol as follows,

$$
\begin{gathered}
u(x, y)=U_{B}(y)+\widetilde{u}(y) \varepsilon e^{i(\alpha x+\omega t)}+O\left(\varepsilon^{2}\right) \\
v(x, y)=\widetilde{v}(y) \varepsilon e^{i(\alpha x+\omega t)}+O\left(\varepsilon^{2}\right) \\
p(x, y)=p_{B}(x)+\widetilde{p}(y) \varepsilon e^{i(\alpha x+\omega t)}+O\left(\varepsilon^{2}\right) \\
C_{i}(x, y)=C_{B i}(y)+\widetilde{C}_{i}(y) \varepsilon e^{i(\alpha x+\omega t)}+O\left(\varepsilon^{2}\right)
\end{gathered}
$$

Using above, the governing equations are decomposed into a base and perturbed parts, the solution of the base part is obtained analytically and that of a perturbed part is obtained numerically. Splitting (8), (10) to (15) and neglecting the higher orders of 
perturbation parameter $\varepsilon$, the base part and perturbed part equations of velocities and concentration are obtained. Assuming the flow to be steady the following set of partial differential equations are derived.

Base part

$$
\begin{gathered}
\frac{\partial^{2} U_{B}}{\partial y^{2}}-M^{2} U_{B}-P_{1} R e+W_{e} a_{2} e^{-\alpha_{c} y}+G \sin \theta=0 \\
\frac{\partial^{2} C_{B i}}{\partial y^{2}}-\beta^{2} C_{B i}=0
\end{gathered}
$$

Perturbed part

$$
\begin{gathered}
\frac{\partial^{2} \widetilde{u}}{\partial y^{2}}+\widetilde{u}\left(\operatorname{Re} a_{0} a_{4}-M^{2}-\alpha^{2}+\operatorname{Re} a_{3} U_{B}\right)-\operatorname{Re} \widetilde{v} \frac{\partial U_{B}}{\partial y}-\operatorname{Re} a_{3} \widetilde{p}=0 \\
\frac{\partial \widetilde{v}}{\partial y}=\widetilde{u} a_{3} \\
\frac{\partial^{2} \widetilde{v}}{\partial y^{2}}-\widetilde{v}\left(a_{3} U_{B}+\alpha^{2}+\operatorname{Re} a_{0} a_{4}\right)=0 \\
\frac{\partial^{2} \widetilde{C}_{i}}{\partial y^{2}}+\widetilde{C}_{i}\left(a_{1} a_{3} U_{B}-a_{2} a_{4}-\alpha^{2}-\beta^{2}\right)-a_{1} \widetilde{v} \frac{\partial C_{B i}}{\partial y}=0
\end{gathered}
$$

Where $P_{1}=\frac{\partial p_{B}}{\partial x}, a_{3}=\alpha C_{B i} \tan (\alpha x+\omega t), a_{4}=w \tan (\alpha x+\omega t)$, with base part boundary conditions,

$$
\begin{gathered}
U_{B}=0, C_{B i}=0, \text { at } y=0 \\
C_{B i}=1, \text { at } y=1 \\
\frac{\partial U_{B}}{\partial y}=-\alpha \sigma\left(U_{B}-U_{p_{B}}\right), \text { at } y=1 \\
\frac{\partial U_{B}}{\partial y}=\alpha \sigma\left(U_{B}-U_{p_{B}}\right), \text { at } y=-1
\end{gathered}
$$

and perturbed part boundary conditions below,

$$
\begin{gathered}
\widetilde{u}=0, \widetilde{v}=0, \widetilde{C}_{i}=1, \text { at } y=0 \\
\widetilde{v}=1, \widetilde{C}_{i}=1, \text { at } y=1 \\
\frac{\partial \widetilde{u}}{\partial y}=-\alpha \sigma\left(\widetilde{u}-\widetilde{u_{p}}\right), \text { at } y=1 \\
\frac{\partial \widetilde{u}}{\partial y}=\alpha \sigma\left(\widetilde{u}-\widetilde{u_{p}}\right), \text { at } y=-1
\end{gathered}
$$

The solution for Base part equation (16) is,

$$
U_{B}=A \cosh [M y]+B \sinh [M y]-a_{5}-a_{6} e^{-\alpha_{c} y}
$$

The value of the constants $\mathrm{A}$ and $\mathrm{B}$ are calculated using boundary conditions (22), (24) and (25) gives $A=a_{5}+a_{6}, B=$ $\frac{-a_{5} \alpha_{c} \cosh \left[\alpha_{c}\right]}{M \cosh [M]}$ and $a_{5}=\frac{P_{1} R_{e}-G \sin \theta}{M^{2}}, a_{6}=\frac{a_{3} W_{e}}{\alpha_{c}{ }^{2}-M^{2}}$. The concentration of air pollutants with and without chemical reaction is discussed in the following subsections. 


\subsubsection{Case 1: Concentration of smog $\left(C_{1}\right)($ when $\beta \neq 0)$}

The base part of $C_{1}$ is $C_{B 1}$ and its solution is obtained from (17) is given by,

$$
C_{B_{1}}=C \cosh [\beta y]+D \sinh [\beta y]
$$

Using boundary conditions (22) and (23) we get, $C=0, D=\frac{1}{\sinh [\beta]}$

The perturbed part equations of $u, v, C_{1}$ are given in (18), (20) and (21) can be rewritten as,

$$
\begin{gathered}
\frac{\partial^{2} \widetilde{u}}{\partial y^{2}}+\widetilde{u}\left(d_{1}+d_{2} \cosh [M y]+d_{3} \sinh [M y]-d_{4} e^{-\alpha_{c} y}\right)-\widetilde{v}\left(d_{6} \sinh [M y]+d_{7} \cosh [M y]+d_{8} e^{-\alpha c y}\right)-d_{5}=0 \\
\frac{\partial^{2} \tilde{v}}{\partial y^{2}}+\tilde{v}\left(d_{9} \cosh [M y]-d_{10} \sinh [M y]-d_{11} e^{-\alpha_{c} y}\right)+d_{12}=0 \\
\frac{\partial^{2} \widetilde{C}_{1}}{\partial y^{2}}+\widetilde{C}_{1}\left(d_{13} \cosh [M y]+d_{14} \sinh [M y]-d_{15} e^{-\alpha c y}-d_{16}\right)-\widetilde{v}\left(d_{17} \sinh [\beta y]+d_{18} \cosh [\beta y]\right)=0
\end{gathered}
$$

Where $d_{1}=\operatorname{Re} a_{0} a_{4}-\operatorname{Re} a_{3} a_{5}-M^{2}-\alpha^{2}, d_{2}=\operatorname{Re} A a_{3}, d_{3}=\operatorname{Re} B a_{3}, d_{4}=\operatorname{Re} a_{3} a_{6}, d_{5}=\operatorname{Re} a_{3} \widehat{p}, d_{6}=\operatorname{Re} A M, d_{7}=$ $\operatorname{Re} B M, d_{8}=\alpha c \operatorname{Re} a_{6}, d_{9}=A a_{3}, d_{10}=B a_{3}, d_{11}=a_{6} a_{3}, d_{12}=a_{0} a_{4} \operatorname{Re}-a_{3} a_{5}+\alpha^{2}, d_{13}=a_{1} a_{3} A, d_{14}=a_{1} a_{3} B, d_{15}=$ $a_{1} a_{3} a_{6}, d_{16}=a_{2} a_{4}+a_{1} a_{3} a_{5}+\beta^{2}+\alpha^{2}, d_{17}=a_{1} C \beta, d_{18}=a_{1} \beta D, d_{19}=a_{1} a_{3} a_{5}+a_{2} a_{4}+\alpha^{2}$.

These perturbed equations are solved numerically subject to the boundary conditions prescribed in (26) to (29). Graphs are plotted for the axial velocity $u$, and concentration of $\operatorname{smog}\left(C_{1}\right)$ using MATHEMATICA.

2.1.2 Case 2: Concentration of Haze $\left(C_{2}\right)$ ( when $\beta \neq 0$ )

If no chemical reaction takes place in the channel then the reaction rate parameter $\beta$ is zero, the corresponding base part of $C_{2}$ is $C_{B 2}$ is given by,

$$
\frac{\partial^{2} C_{B 2}}{\partial y^{2}}=0
$$

The solution of $C_{B 2}$ is got by integrating the above equation twice so,

$$
C_{B 2}=E y+F
$$

The constants $E, F$ are calculated using boundary conditions described in (22), (23) which are given by $E=0, F=1$. The real perturbed equation of concentration without chemical reaction is,

$$
\frac{\partial^{2} \widetilde{C}_{2}}{\partial y^{2}}+\widetilde{C_{2}}\left(a_{1} a_{3} U_{B}-a_{2} a_{4}-\alpha^{2}\right)-a_{1} \widetilde{v} \frac{\partial C_{B 2}}{\partial y}=0
$$

and (37) can be rewritten as

$$
\frac{\partial^{2} \tilde{C}_{2}}{\partial y^{2}}+\tilde{C}_{2}\left(d_{13} \cosh [M y]+d_{14} \sinh [M y]-d_{15} e^{-\alpha c y}-d_{19}\right)-\tilde{v}\left(a_{1}\right)=0
$$

Equation (38) is solved numerically for $\widetilde{C}_{2}$ and a graphical solution is obtained for $C_{2}$ using MATHEMATICA.

\section{Results and Discussion}

The velocity profile for different values of angle of inclination $(\theta)$, Hartmann number $(M)$, electric number $(W e)$ and gravity parameter $(\mathrm{G})$ are displayed in Figures 2, 3, 4 and 5. The velocity vectors are shown to get a better insight of the flow. Figure 2 depicts that increasing the angle of inclination velocity decreases. But by increasing the values of Hartmann number, electric number and gravity parameter, the velocity increases respectively. The effects of the concentration with and without chemical reaction on various parameters are described in the proceeding sub-sections. 


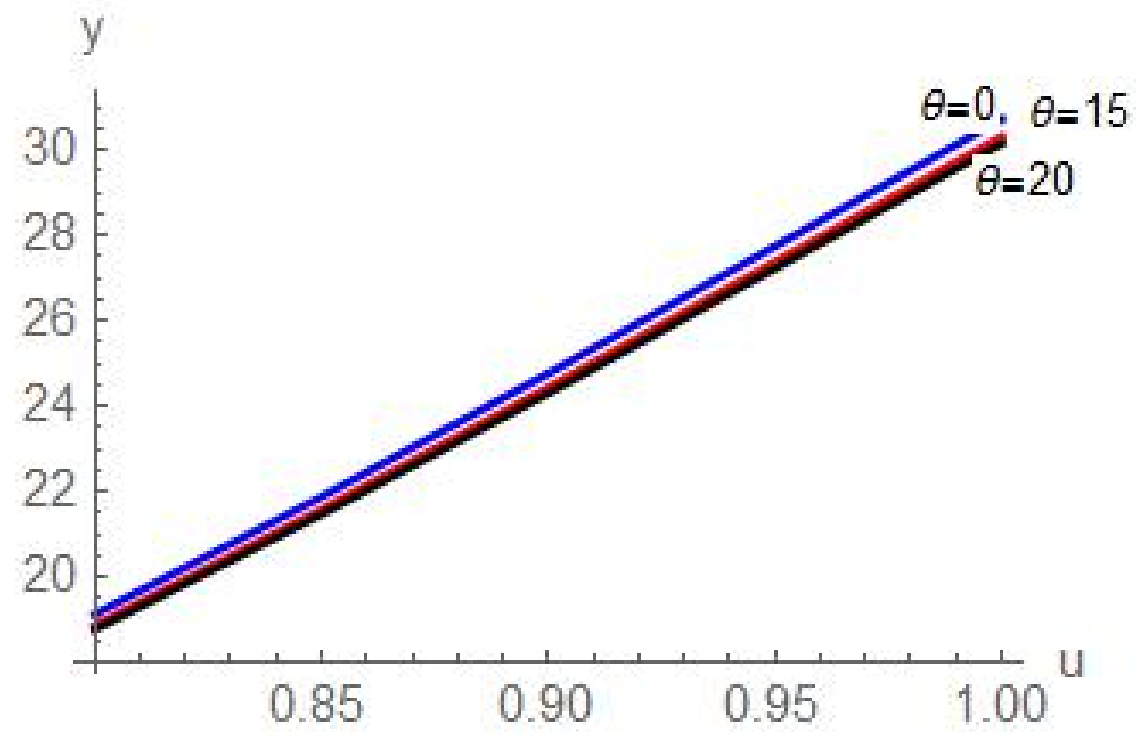

Fig 2. Velocity profile for different values of angle of inclination

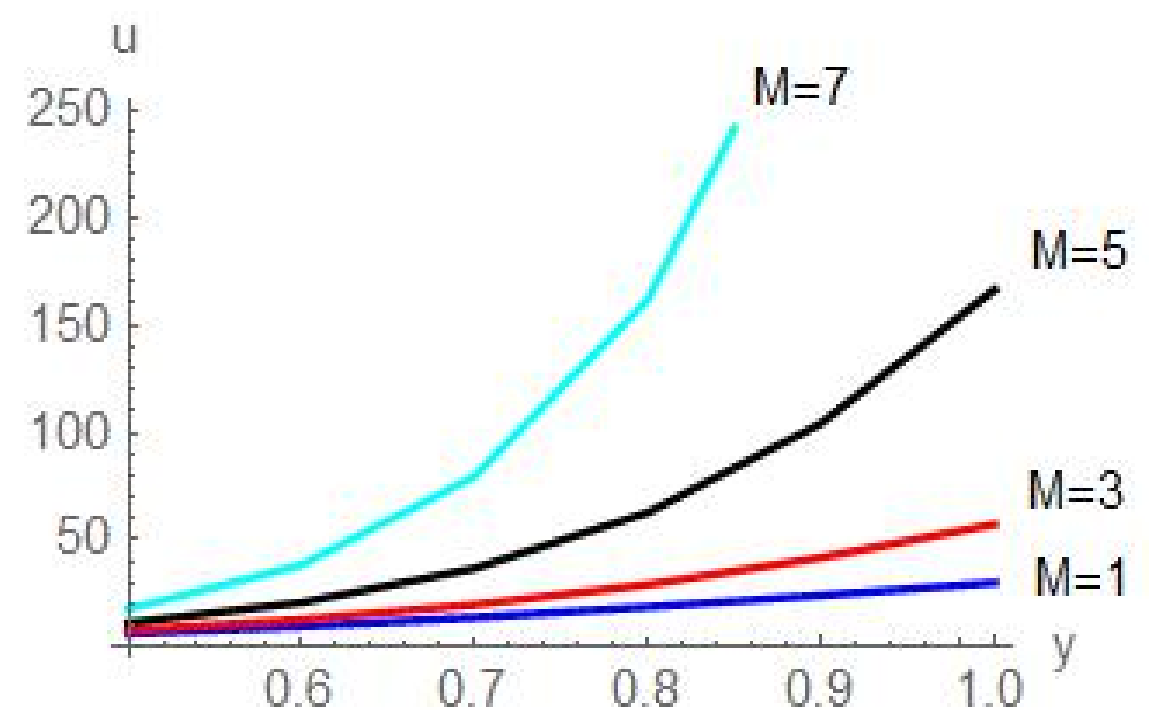

Fig 3. Variation of velocity for some values of Hartmann number 


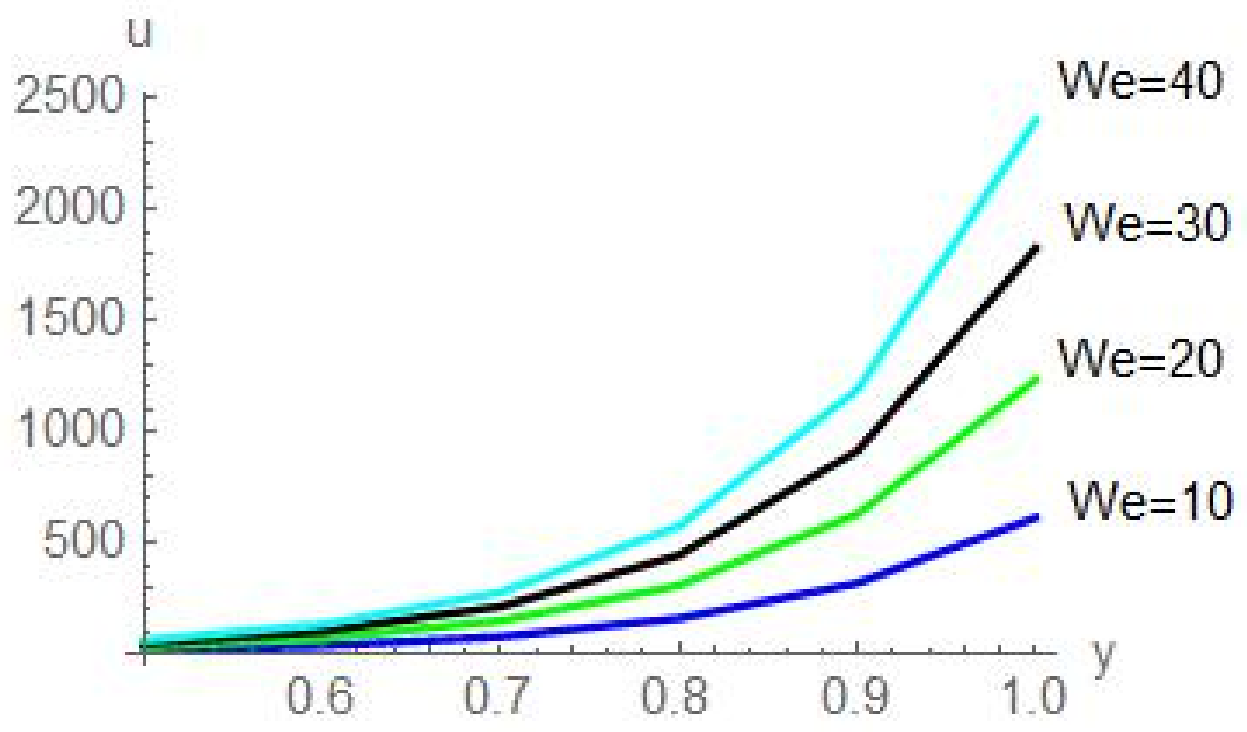

Fig 4. Plots of velocity for various values of electric number

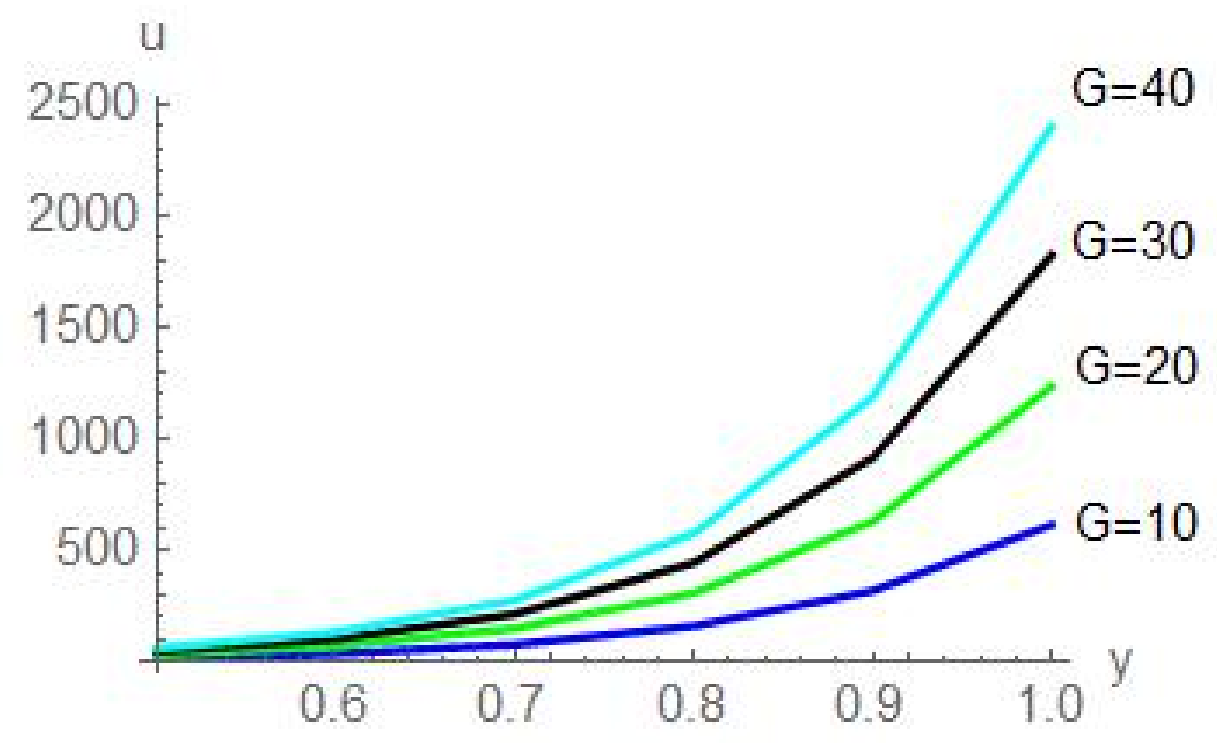

Fig 5. Velocity profile for some values of gravity parameter 


\subsection{Effects of concentration of smog}

Figure 6 depicts that increasing the rate of the reaction, smog concentration increases. Figure 7 tells that by increasing the gravity parameter the smog concentration increases. Also, for the small value of the gravity parameter, the smog concentration is less. Figure 8 predicts that while increasing the Hartmann number, the smog concentration increases. But in the absence of a magnetic field, the concentration of smog is low. In Figure 9, plots clearly indicate that increasing electric number concentration of smog increases. In the absence of an electric field, the concentration of smog is less.

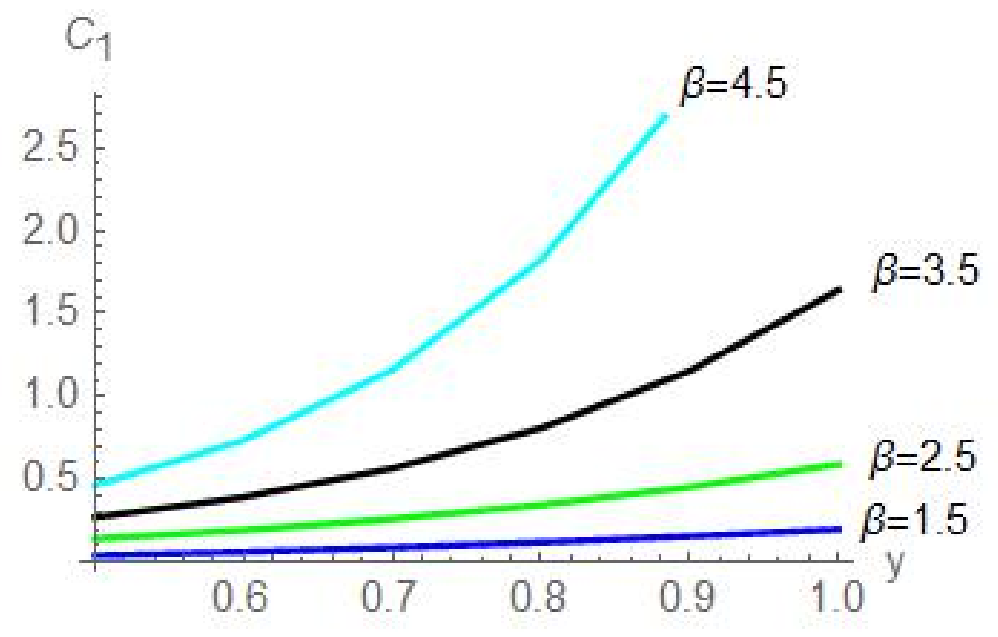

Fig 6. Plots of smog concentration for various values of reaction rate parameter

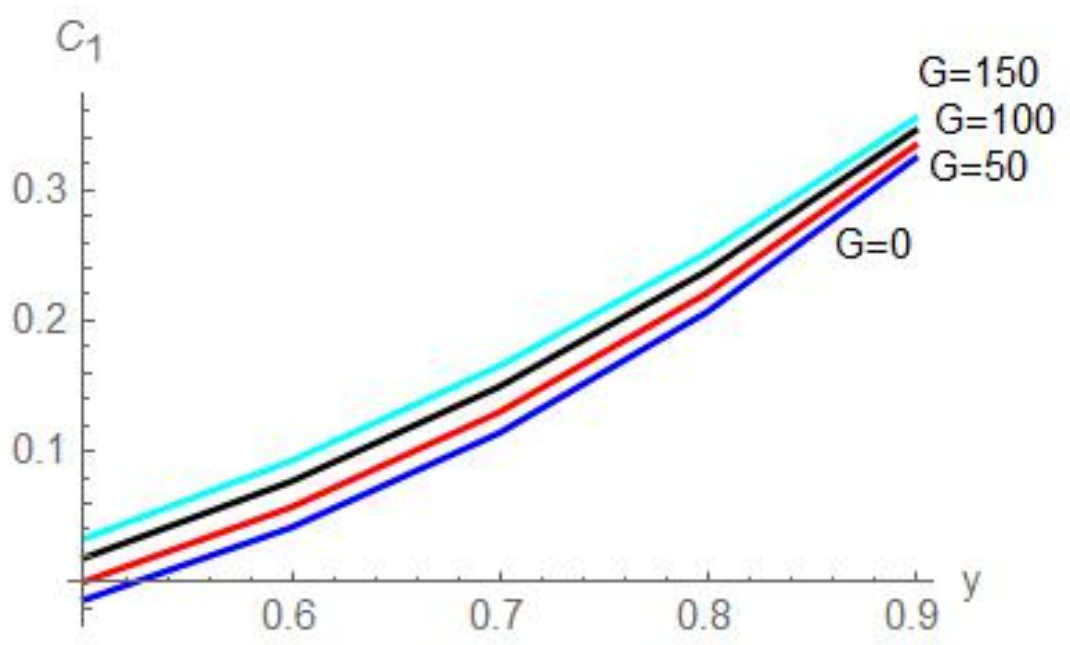

Fig 7. Plots of smog concentration for different values of gravity parameter 


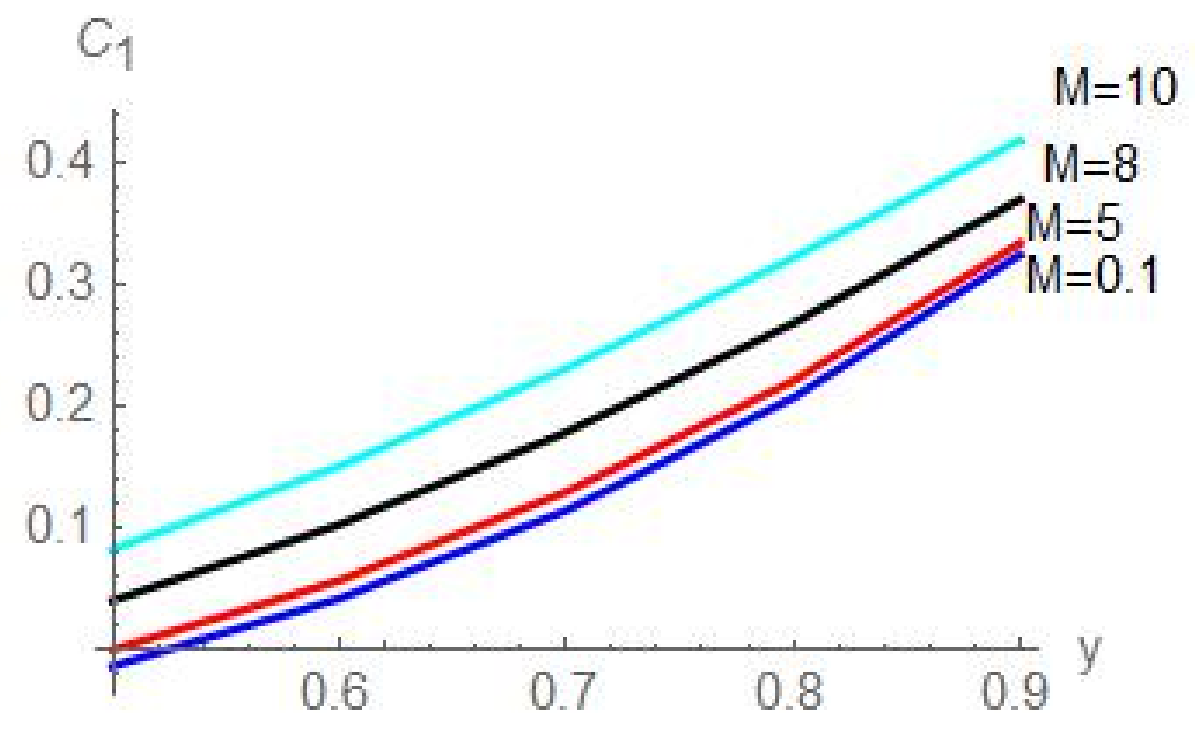

Fig 8. Smog concentration plots for various values of Hartmann number

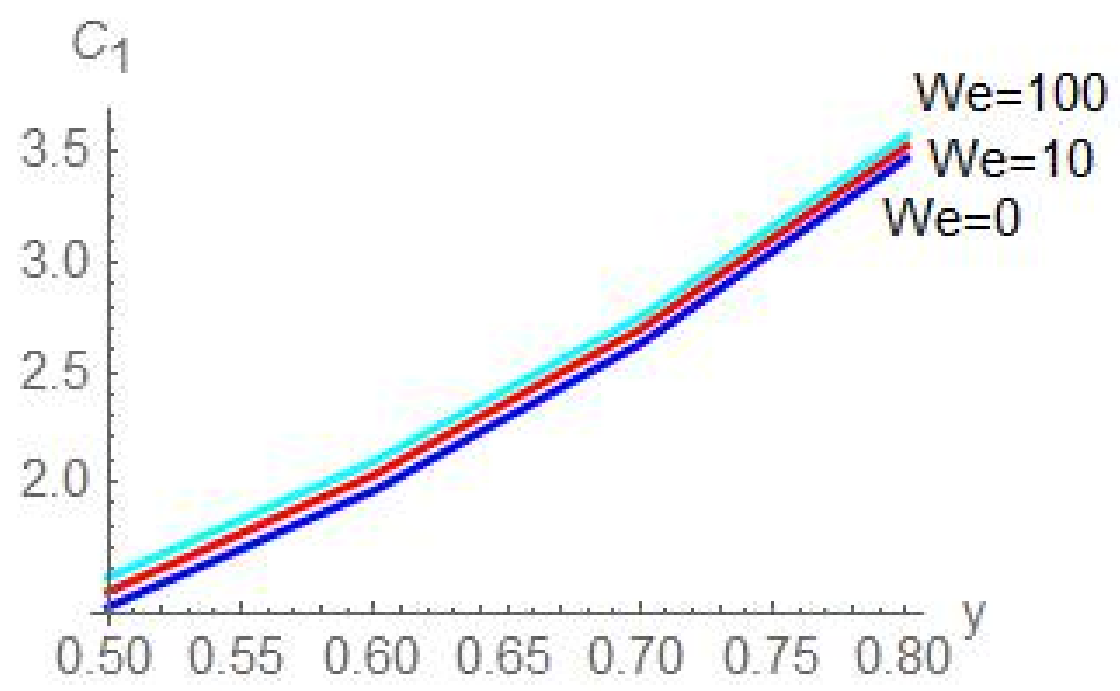

Fig 9. Plots of smog concentration for some values of an electric number 


\subsection{Effects of concentration of Haze}

Figure 10 depicts that increasing the gravity parameter, haze concentration increases, but in the absence of gravity haze concentration is less. Figure 11 shows that if the effects of the magnetic field increase the haze concentration increases. Also in the absence of a magnetic field, the haze concentration is low. But results vary in Figure 12 which shows that increasing the effects of an electric field, the concentration of haze decreases. Also in the absence of an electric effect, the haze concentration becomes high.

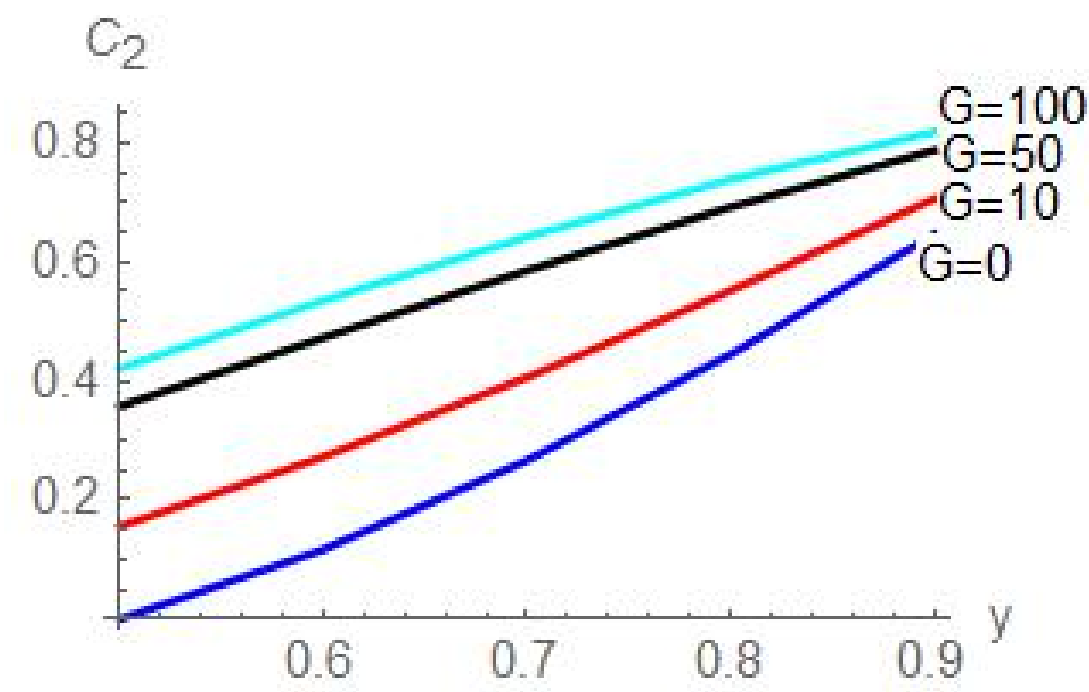

Fig 10. Haze concentration plots for different values of gravity parameter

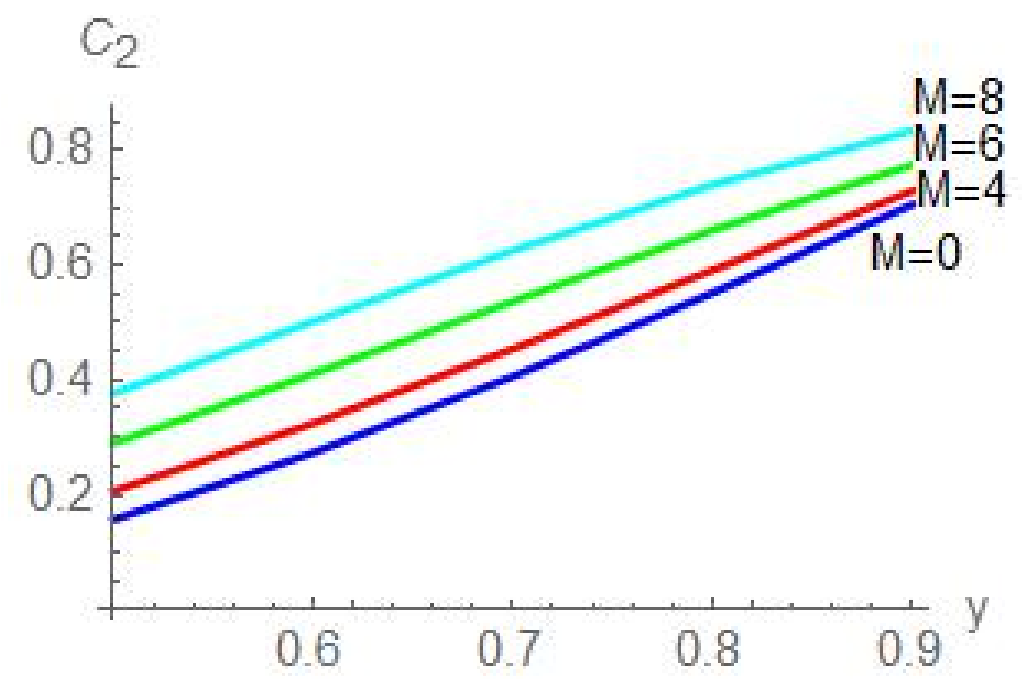

Fig 11. Plots of haze concentration for some values of Hartmann number 


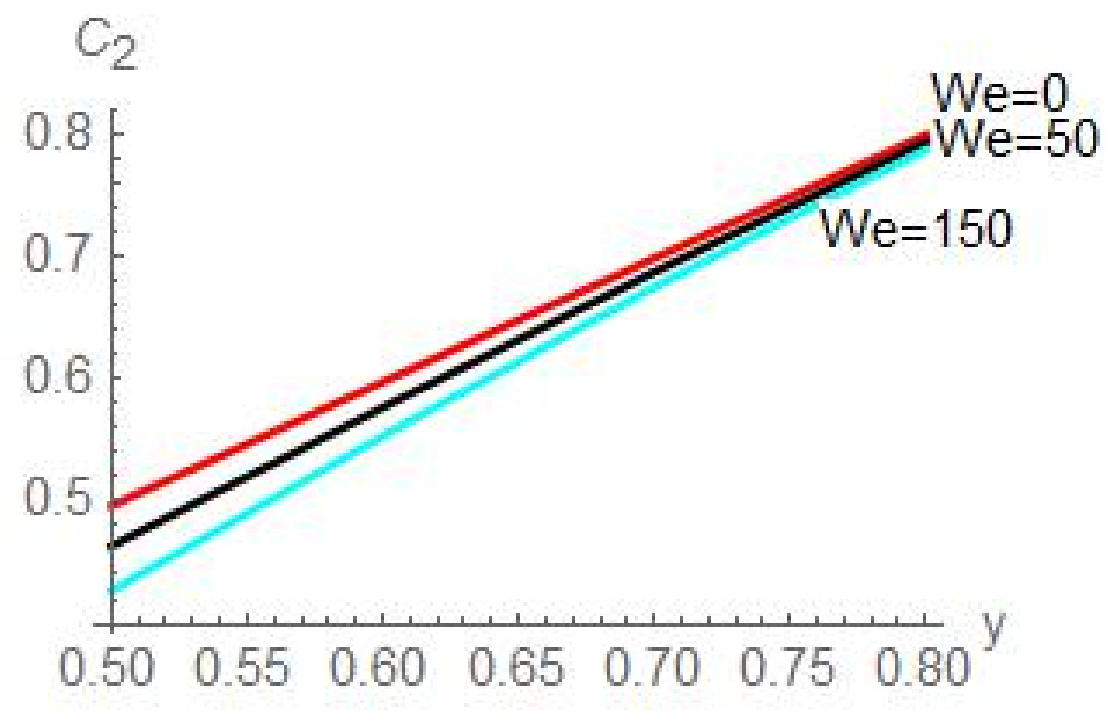

Fig 12. Haze concentration for various values of the electric number

Hence, in the absence of both electric and magnetic field effects smog concentration declines. Also in the absence of magnetic field effects, haze concentration reduces. By enhancing electric field effects haze concentration decreases. Thus, there is no considerable outcome of magnetic effects to reduce the concentration of air pollutants in an inclined channel.

Comparing the results with ${ }^{(14)}$ where dispersion coefficient of aerosols in the horizontal channel together with electric field effects and the porous boundary is investigated and concludes that the electric field and chemical reaction enhances the dispersion of aerosols.

$\mathrm{In}^{(15)}$ the concentration of smog and haze of atmospheric fluid are calculated in the horizontal channel together with the effect of electric field, magnetic field and porous boundaries. The results of concentration in atmospheric fluid conclude that the concentration of smog is reduced by enhancing both electric and magnetic fields and haze concentration is minimized by improving the magnetic effect and reducing the effects of an electric field.

Comparing the obtained results of the present study with the previous studies in the horizontal channel, it gives a clear picture that results differ in exactly opposite regarding electric and magnetic field effects.

\section{Conclusion}

As an effort to reduce damage caused by aerosol the present mathematical model discusses air visibility issues through the effect of smog and haze concentration in an inclined channel bounded by porous beds. The concentration of smog and haze are obtained using the perturbation technique together with the effects of an electric and magnetic field. The numerical results were presented graphically and are extensively discussed for reasonable values of dimensionless parameters. It is concluded that in an inclined channel, enhancing the electric field effects, the haze concentration is reduced and also decreasing both electric and magnetic field effects, the concentration of smog declines. There are no considerable effects of porous boundaries in the model.

\section{References}

1) Lakshminarayanachari K, Pai KLS, Prasad MS, Pandurangappa C. A two dimensional numerical model of primary pollutant emitted from an urban area source with mesoscale wind, dry deposition and chemical reaction. Atmospheric Pollution Research. 2013;4(1):106-116. Available from: https: //dx.doi.org/10.5094/apr.2013.011.

2) Junji C. The importance of Aerosols in the Earth system: Science and engineering perspectives. Aerosol Science and Engineering. 2017;1:1-6.

3) Meenapriya P. Electric field effects on dynamic behavior of atmospheric aerosols. Journal of Emerging Technologies and Innovative Research. 2018;5(11):350-359. 
4) Meenapriya P. Effect of magnetic field on scattering of dust particles with chemical reaction in the atmosphere. International Journal of Mathematical Sciences. 2019;18(1-2):145-154.

5) Dettman JW. Perturbation techniques in related differential equations. Journal of Differential Equations. 1973;14(3):547-558. Available from: https: //dx.doi.org/10.1016/0022-0396(73)90066-1.

6) Nayfeh AH. Introduction to perturbation technique. New York. Wiley publications. 1981.

7) Malashetty MS, Umavathi JC, Kumar JP. Two fluid flow and heat transfer in an inclined channel containing porous and fluid layer. Heat and Mass Transfer. 2004;40(11):871-876. Available from: https://dx.doi.org/10.1007/s00231-003-0492-2.

8) Majlesara M, Salmanzadeh M, Ahmadi G. A model for particles deposition in turbulent inclined channels. Journal of Aerosol Science. 2013;64:37-47. Available from: https://dx.doi.org/10.1016/j.jaerosci.2013.06.001.

9) Reddappa B, Sreenadh S, Naidu KK. Convective coquette flow of a Jeffrey fluid in an inclined channel when the walls are provided with porous lining. Advances in Applied Science Research. 2015;6(12):69-75.

10) Rathod VP, Manikrao N, Devindrappa L. Peristaltic flow of a couple stress fluid in an inclined channel under the effect of magnetic field by Adomian decomposition method ( X - ct )) b. Advances in Applied Science Research. 2016;7(2):81-91.

11) Ramakrishnan K, Shailendhra K. Hydromagnetic flow through uniform channel bounded by porous media. Applied Mathematics and Mechanics. 2011;32(7):837-846. Available from: https://dx.doi.org/10.1007/s10483-011-1463-7.

12) Murty PSR, Prakash GB. MHD Two-Fluid Flow and Heat Transfer between Two Inclined Parallel Plates in a Rotating System. International Scholarly Research Notices. 2014;2014:1-11. Available from: https://dx.doi.org/10.1155/2014/256898.

13) Beavers GS, Joseph DD. Boundary conditions at a naturally permeable wall. Journal of Fluid Mechanics. 1967;30(1):197-207. Available from: https://dx.doi.org/10.1017/s0022112067001375.

14) Rudraiah N, Devaraju N, Ng CO. Electrohydrodynamic Dispersion of Deformable Aerosols in the Presence of an Electric Field and Chemical Reaction Using Taylor Dispersion Model. Journal of Hydrodynamics. 2011;23(2):247-257. Available from: https://dx.doi.org/10.1016/s1001-6058(10)60110-2.

15) Meenapriya P, Maheswari KU, Ratchagar NP. Effects of Concentration of Air Pollutants in the channel bounded by porous beds. Advances in Mathematics: Scientific Journal. 2020;9(4):1699-1711. Available from: https://dx.doi.org/10.37418/amsj.9.4.26. 